\title{
Clinical and genetic investigation of ichthyosis in familial and sporadic cases in south of Tunisia: genotype-phenotype correlation
}

\author{
Mariem Ennouri ${ }^{*}$, Andreas D. Zimmer ${ }^{2}$, Emna Bahloul $^{3}$, Rim Chaabouni ${ }^{3}$, Slaheddine Marrakchi ${ }^{3}$, \\ Hamida Turki ${ }^{3}$, Faiza Fakhfakh', Noura Bougacha-Elleuch ${ }^{1}$ and Judith Fischer ${ }^{2}$
}

\begin{abstract}
Background: Ichthyosis is a heterogeneous group of Mendelian cornification disorders that includes syndromic and non-syndromic forms. Autosomal Recessive Congenital Ichthyosis (ARCI) and Ichthyosis Linearis Circumflexa (ILC) belong to non-syndromic forms. Syndromic ichthyosis is rather a large group of heterogeneous diseases. Overlapping phenotypes and genotypes between these disorders is a major characteristic. Therefore, determining the specific genetic background for each form would be necessary.

Methods: A total of 11 Tunisian patients with non-syndromic ( 8 with ARCI and 2 with ILC) and autosomal syndromic ichthyosis (1 patient) were screened by a custom Agilent HaloPlex multi-gene panel and the segregation of causative mutations were analyzed in available family members.

Results: Clinical and molecular characterization, leading to genotype-phenotype correlation in 11 Tunisian patients was carried out. Overall, we identified 8 mutations in 5 genes. Thus, in patients with $A R C l$, we identified a novel (c.118T > C in NIPAL4) and 4 already reported mutations (c.534A > C in NIPAL4; C.788G > A and C.1042C > T in TGM1 and c.844C > T in CYP4F22). Yellowish severe keratoderma was found to be associated with NIPAL4 variations and brachydactyly to TGM1 mutations. Two novel variations (c.5898G > C and c.2855A>G in ABCA12) seemed to be features of ILC. Delexon13 in CERS3 was reported in a patient with syndromic ichthyosis.
\end{abstract}

Conclusions: Our study further extends the spectrum of mutations involved in ichthyosis as well as clinical features that could help directing genetic investigation.

Keywords: Ichthyosis, TGM1, NIPAL4, CYP4F22, CERS3, ABCA12

\section{Introduction}

Ichthyosis is a heterogeneous group of Mendelian cornification disorders that includes syndromic and nonsyndromic forms. Autosomal Recessive Congenital

\footnotetext{
*Correspondence: mariamnouri1992@gmail.com

${ }^{1}$ Laboratory of Molecular and Functional Genetics, Faculty of Sciences

of Sfax, Sfax University, Sfax University, Street of Soukra km 4, BP

1171-3000, Sfax, Tunisia

Full list of author information is available at the end of the article
}

Ichthyosis (ARCI) and Ichthyosis Linearis Circumflexa (ILC) belong to non-syndromic forms. ARCI is characterized by abnormal desquamation over the whole body due to a dysfunctional skin permeability barrier and altered lipid composition. It is a rare skin disease affecting around 1 in 200,000 individuals $[1,2]$.

ARCI defines three major clinical subtypes with varying severity degrees, including the spectrum of Congenital Ichthyosiform Erythroderma (CIE), Lamellar Ichthyosis 
(LI) and Harlequin Ichthyosis (HI). HI is the most severe form of inherited ichthyosis and can be fatal. The clinical features of this subgroup include thick, plate-like scales with severe ectropion, flattening of the ears and eclabium $[3,4]$. LI form is a milder phenotype, characterized by a thick dark/brown scale covering the majority of the body and palmoplantar keratoderma [5]. LI patients are usually born as collodion babies. CIE phenotype is rather characterized by fine, white scales and erythroderma. Like LI, CIE babies are born as collodion. ILC is a rare form of ichthyosis characterized by polycyclic patches bordered by a double-edged scale.

Beside clinical heterogeneity, a large genetic heterogeneity was described in ichthyosis. Thirteen genes, encoding proteins essential for skin barrier formation [6, 7] have been involved: TGM1 (MIM:190195) [7], NIPAL4 (MIM:609383) [8], ALOXE3 (MIM: 607206) [9], ABCA12 (MIM: 607800) [10], SDR9C7 (MIM: 609769) [11], PNPLA1 (MIM: 612121) [12], SULT2B1 (MIM: 604125) [13], CYP4F22 (MIM: 611495) [14], CERS3 (MIM: 615276) [15], ALOX12B (MIM: 603741) [16], LIPN (MIM: 613924) [17], SLC27A4(MIM: 604194) [18], and ST14 (MIM: 606797) [19].

A major characteristic of ARCI is partially overlapping phenotypes and genotypes between LI and CIE [20] (Table 1). Therefore, it would be difficult to determine the specific genetic background for each form. However, involvement of particular genes in specific forms was proposed.

The current study focused on 11 newly recruited Tunisian patients affected with either non-syndromic ichthyosis forms (8 with ARCI: 4 with CIE and 4 with LI; 2 with ILC) or autosomal syndromic ichthyosis (1 patient). We tried to highlight some clinical features related to specific genes that could facilitate genetic diagnosis. We reported three novel missense mutations in two different consanguineous families, as well as 5 known disease-causing variants in 4 different genes in the remaining subjects. In silico analysis was performed in order to predict the effect of novel mutations on each corresponding protein level.

\section{Methods}

\section{Patients}

A total of 11 patients with different ichthyosis phenotypes were enrolled. Among them, five belonged to two consanguineous families: A (A3, A4 and A7) and F (F1 and F2). The other six cases (M1, B1, C1, Y1, K1 and E1) were sporadic. Ten out of 11 patients were affected with non-syndromic ichthyosis (8 with ARCI: 4 with CIE and 4 with LI; 2 with ILC). Only one patient suffered from autosomal syndromic ichthyosis.

The study protocol conforms to the approval of the local ethical committee of CHU Hedi Chaker, Sfax, in compliance with the Declaration of Helsinki. Written informed consent was obtained from all study participants or the parents for minor children.

\section{DNA extraction}

Total genomic DNA was extracted from patients and available members' family using the phenol-chloroform standard procedure [21]. The DNA concentration was

Table 1 Summary table of clinical signs and genetic background associated with ichthyosis forms (LI, CIE and ILC) [3]

\begin{tabular}{llll}
\hline Characteristics & Ichthyosis form & CIE & ILC \\
\cline { 2 - 4 } & LI & AR & AD \\
Mode of inheritance & AR & At birth & At birth or neonatal \\
Onset & At birth & CIE or less frequently mild collodion & CIE \\
Initial clinical presentation & Collodion membrane with ectropion and & membrane & Localized serpiginous, migratory \\
Distribution of scaling & Gelabium; less frequently CIE & Generalized; focally pronounced scaling & Fine \\
Scaling type & possible & possible & White \\
Scaling color & Coarse and large (plate like) & Fine & Variable \\
Erythema & Brownish or dark & White or gray & No \\
Palmoplantar involvement & Variable, less pronounced & Variable, often pronounced & \\
& mild keratoderma & NIPAL4: pronounced keratoderma & \\
Scalp abnormalities & TGM1: frequent palmoplantar involvement & TGM1: frequent palmoplantar involvement & No
\end{tabular}

L/ lamellar ichthyosis; CIE congenital ichthyosiform erythoderma; ILC ichthyosis linearis circumflexa; $A R$ autosmic recessive; $A D$ autosomic dominant 
measured with a Qubit 2.0 fluorometer (Life Technologies, Carlsbad, CA, USA).

\section{Next generation sequencing}

Next generation sequencing (NGS) was performed using a custom Agilent HaloPlex multi-gene panel with 32 ichthyosis linked genes comprising a target size of $\sim 64 \mathrm{kbp}$. The average sequencing depth was $420.3 \mathrm{x}$. A rate of $97.6 \%$ of the target position had at least $20 \times$ in depth. Sequencing was performed on an Illumina MiSeq sequencer using MiSeq reagent kit v2 $(2 \times 150 \mathrm{bp})$. Reads were aligned using bwa (v. 0.7.17) [22] against human hg19, and after recalibration, realignments, and genotype calling with gatk (gatk38 nightly-2017-12-06-1) [23], freebayes (v1.2.0-dirty) [24], vardict (v20180621) [25], bcftools (v.1.9) [26]. Variants were annotated using ANNOVAR [27], Variant Effect Predictor [28] and custom scripts.

\section{Sanger sequencing}

PCR amplification was performed using a thermal cycler GeneAmp PCR System 2720 (Applied Biosystems, Foster City, CA), in a final volume of $15 \mu \mathrm{l}$ using $100 \mathrm{ng}$ DNA, $10 \mu \mathrm{M}$ of each primer, $2 \mathrm{mM}$ dNTP, $10 \times$ PCR buffer, $1 \mathrm{U}$ of Taq DNA polymerase (Qiagen) and Q-solution. Primers were generated with primer3 and corresponding sequences are mentioned in Table 2.

PCR products were sequenced using Applied biosystem (HITACHI 3500XL analyzer). Sequences were analyzed, using Bio Edit Program and compared with wild type sequences: NIPAL4 (ENST00000311946.7) and ABCA12 (ENSG00000141527.18) by BLAST online software.

\section{Bioinformatic predictions}

Protein sequence alignment across species was performed using CLUSTALW and PolyPhen2 software. The potential functional impact of p.(M63T) mutation on NIPA4 and ABCA12 proteins was predicted using PolyPhen2, SIFT, Mutation Taster, Panther and CADD software.

\section{Results}

\section{Clinical analysis}

The current study included 11 ichthyosis patients with 3 phenotypes. Among them, five belonged to two consanguineous families: A (A3, A4 and A7) and F (F1 and F2). The other six cases (C1, M1, B1, Y1, K1 and E1) were sporadic (Table 3).

All three patients from A family, were born as collodion babies. With age, A3 and A4 evolved to CIE, while A7 developed LI. At the age of one year, cutaneous examination of patient A4 showed generalized white fine scales with an erythematous underlying skin. Her brother (A3) displayed at the age of 3 years larger scales on the forehead and limbs, while the scales were fine and brown on the trunk on an erythematous skin. However, their first cousin (A7), who was affected with LI demonstrated darker, thicker and plate-like scales all over the body, without erythema (Fig. 1a). Patients with CIE (A3 and A4), showed sparse light brown hair compared to A7 and to the rest of the family members. Particularly, the folds were involved in A3 and A7, while hyperlinearity of the soles was noted only in A3. Patient A7 had a diffuse but not severe plantar keratoderma developed at the age of 6 years, while palms and soles were spared in patient A4.

The F family included 2 patients (F1 and F2). The proband $F 1$, aged 35 years, had a similar phenotype to her 4 year-old daughter (F2). Both patients presented multiple polycyclic erythematous and squamous plaques, with brownish fine scales (Fig. 1b). Those lesions were located on the trunk in patient $\mathrm{F} 1$, and in the forearms, elbows and inguinal folds in the patient F2. This latter had cutaneous xerosis at birth. No ectropion, no alopecia and no ear malformation were noted in both patients.

C1 patient, who was 34 years old, had CIE with a mild erythema of the skin and small white scales sparing the folds. He presented a severe yellowish keratoderma of the palms and soles with a pseudo-ainhum and reduced fingers mobility (Fig. 1c, d). These aspects of extremities were very similar to those seen in Mal de Meleda disease. There was no ectropion and ears were normal.

M1 patient, aged of 74 years and also affected with CIE, showed mild erythroderma with fine white scales

Table 2 Primers' sequences used for genes amplification

\begin{tabular}{lll}
\hline Gene/exon & Primers sequences & Reverse \\
\cline { 2 - 3 } & Forward & 5'CCTGGGAGGAGAGGATGC3' \\
\hline NIPAL4/exon1 & 5'CATCTAGGTCCCCTGTACTC3' & 5'TGTATCTGTAGGAAGCACATTCA3' \\
ABCA12/exon18 & 5'GGCTGTGCTGTCTAATCCTAGC3' & 5'GGTGTGAGCCACTGTACCTG3' \\
\hline
\end{tabular}




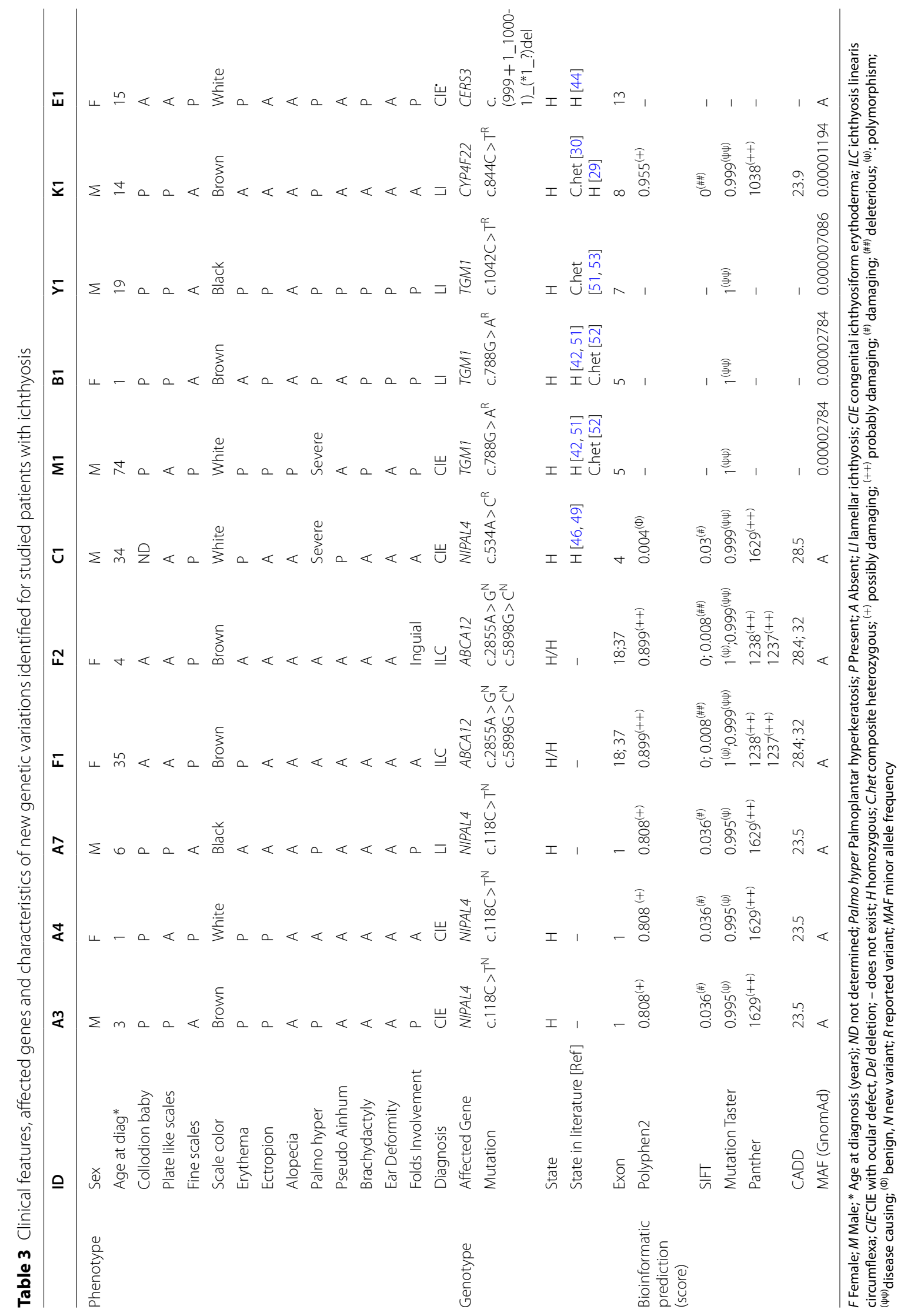




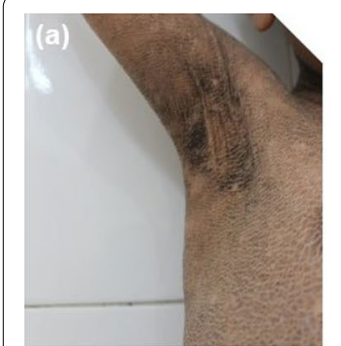

A7

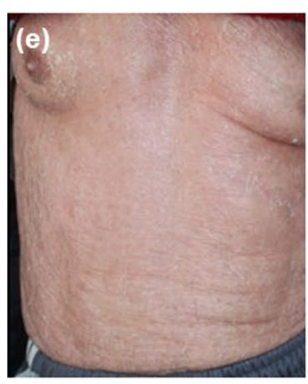

M1

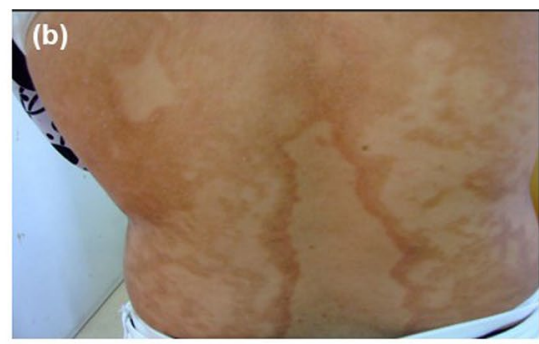

F1

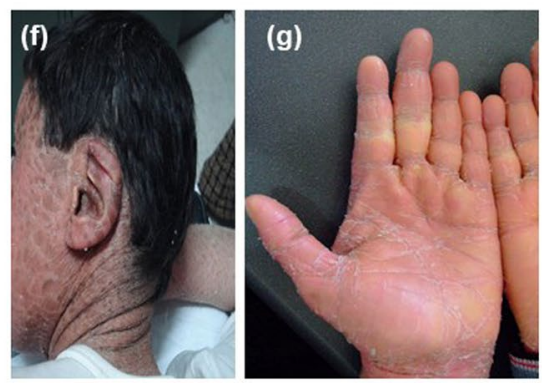

Y1

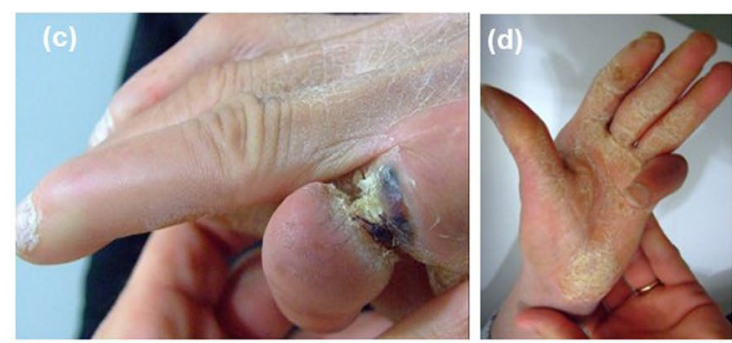

C1

Fig. 1 Clinical features of some studied patients. a Dark, thick and plate-like scales on the axilla of patient A7 (NIPAL4); $\mathbf{b}$ polycyclic erythematous and squamous plaques, with brownish fine scales on the trunk of patient F1 (ABCA12); c pseudoainhum of the right finger in patient C1 (NIPAL4), $\mathbf{d}$ a yellowish palmar keratoderma in patient C1 (NIPAL4); e skin of the trunk of patient M1 showing ichthyosiform erythroderma with fine, white scales (TGM1); f Patient Y1 displayed large brown plate-like scales on the face and neck with adhered ear (TGM1); $\mathbf{g}$ hands of patient Y1 showing diffuse palmar keratoderma and brachydactyly (TGM1)

covering the whole body (Fig. 1e). He was born as a collodion baby and revealed, later, bilateral ectropion and diffuse palmoplantar keratoderma (PPK) or palmoplantar hyperlinearity. The folds were involved and alopecia was particularly noted. Brachydactyly with long palm and short fingers was also noted. Moreover, M1 patient suffered from painful palmar fissures and necrosis of the distal pulp of the second right finger and the third left finger secondary to repetitive infections.

On the other hand, B1, Y1 and K1 had LI phenotype. B1 patient aged one year, presented brown large plate like scales covering the entire body without erythema, while Y1 patient had black large plate like scales covering the entire body including the folds on an erythematous skin. Y1 displayed large brown plate-like scales on the face and neck with adhered ear (Fig. 1f). His hands showed diffuse palmar keratoderma (Fig. 1g). Both patients (B1 and Y1) were born as collodion babies and revealed, later, bilateral ectropion and diffuse PPK or palmoplantar hyperlinearity. The folds were involved and ears deformity was seen in both patients. Brachydactyly with long palm and short fingers was also noted. K1, aged 14 years, was born as a collodion baby and developed, later, large brown scales of the front, the trunk and proximal parts of the limbs. The scales became smaller on the legs and forearms. Large folds were spared. Mild palmoplantar keratoderma with hyperlinearity characterized the extremities.
No ectropion, no nails abnormalities, no alopecia and no ears malformation were reported.

Finally, E1 patient affected with syndromic ichthyosis (CIE with ocular defect) presented mild erythematous skin. Scales were white and fine on the face, the trunk and the upper members, while they were thicker and larger on the knees and the legs. White scales were also located on the dorsal aspect of the hands and feet and in the folds. Mild palmoplantar keratoderma and brachydactyly were also observed. There was no ectropion and ears were normal. Interestingly, ophthalmological examination revealed bilateral myopia and microspherophakia.

\section{Molecular analysis}

Using a multi-gene panel, we identified eight diseasecausing variants in five different genes (NIPAL4, TGM1, CYP4F22, ABCA12 and CERS3) (Table 3).

Mutational analysis of NIPAL4 gene revealed two different germ line missense mutations in homozygous state in A family and $\mathrm{C} 1$ patient. The first one is a novel mutation (c.188T > C; ENST00000311946.7) in exon 1, which substitutes a methionine to a threonine (p.Met63Thr; ENSP00000311687.7). It was reported in family A affected members (A3, A4 and A7), with two ARCI phenotypes (CIE and LI). To confirm the segregation of this mutation and its association with ARCI, Sanger sequencing was performed in all available family members and 
control population. This mutation was in heterozygous state for parents and absent in unaffected members and control individuals (Fig. 2a).

The second disease-causing variant (c.534A >C; ENST00000311946.7) occurred in exon4 of NIPAL4 gene and substituted glutamic to aspartic amino acid (p.Glu178Asp; ENSP00000311687.7). It was identified in a CIE patient ( $\mathrm{C} 1,34$ years old) who presented a mild erythema of the skin, with small white scales sparing the folds.

Two novel missense mutations were detected at the homozygous state: c.5898G >C (ENST00000389661.4) (p.Glu1966Asp; $\quad$ ENSP00000374312.4) and c.2855A > G (ENST00000389661.4) (p.Tyr952Cys; ENSP00000374312.4) in ABCA12 gene in family $\mathrm{F}$ (F1 and F2 patients) with ILC (Fig. 2c). Segregation analysis confirmed the presence of these variations at the heterozygous state in the patient's father. At the beginning, Netherton syndrome and Erythrokeratoderma variabilis phenotypes were suspected, but no hair shaft abnormalities were found and no mutations in SPINK5 and EKV genes (GJB2, GJB3, GJB4, GJB6 and KDSR) were identified (data not shown).

In three patients with non-syndromic ichthyosis (M1, B1 and Y1), the involved gene was TGM1 with two different homozygous nonsense germline mutations (Table 3). Interestingly, both variations were described in gnomAD database in only heterozygous state. The most frequent mutation in this gene was c.788G > A (ENST00000206765.6) in exon 5 (p.Trp263X; ENSP00000206765.6) detected in M1 and B1 affected with CIE and LI respectively. The second recurrent disease-causing variant in TGM1 gene was c. $1042 \mathrm{C}>\mathrm{T}$ (ENST00000206765.6) located in exon 7 (p.Arg348X; ENSP00000206765.6) and detected in patient Y1 aged 19 years, who showed LI phenotype.

K1 patient with LI had rather a single homozygous missense mutation (c.844C $>$ T; ENST00000269703.3) in exon 8 which substitutes arginine to tryptophan (p.Arg282Trp; ENSP00000269703.1) in CYP4F22 gene. This variation was also reported only in heterozygous state in gnomAD database.

We also identified a homozygous deletion in exon 13 of CERS3 gene in a 15-year old patient (E1) affected with syndromic ichthyosis (CIE with ocular defect).

\section{Bioinformatic prediction}

As mentioned in Table 3, and according to our in silico study, all novel missense variants were classified as damaging. Thus, no variant was expected to be a benign

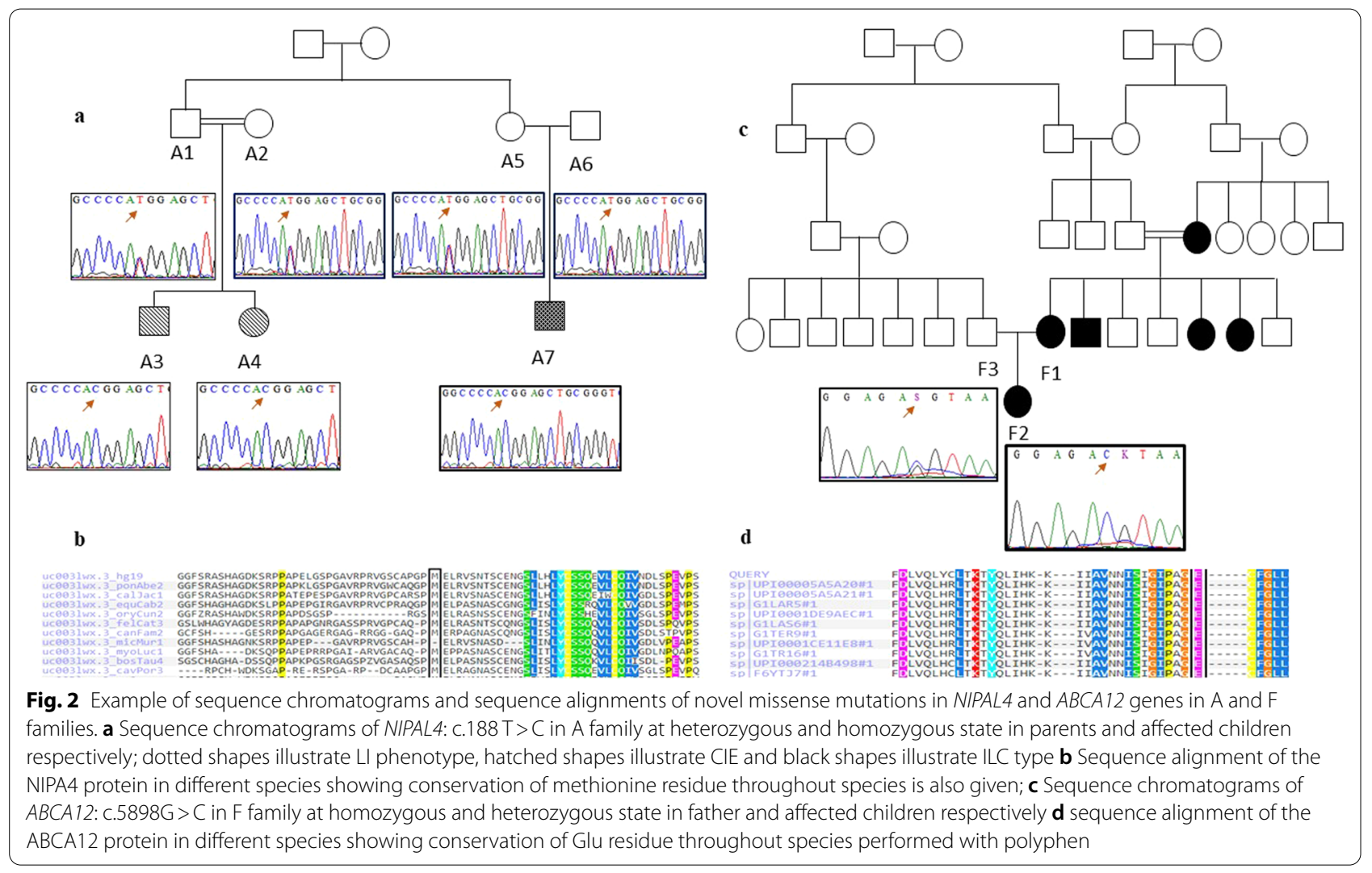


polymorphism using Polyphen2, SIFT, Mutation Taster, Panther and CADD together for the following mutations: p.Met63Thr in NIPAL4 gene and p.Glu1966Asp, p.Tyr952Cys in $A B C A 12$ gene. We have to mention that all new variants were not reported before in the gnom $A D$ database.

Moreover, multiple alignments of corresponding proteins showed that these residues were located within a highly conserved region (Fig. 2b, d).

\section{Discussion}

We collected detailed phenotypic data from 11 Tunisian patients with different ichthyosis forms. In order to identify causative gene(s) mutation(s), we used a custom multi-gene panel. One of the most important aspects of this panel is its ability to be easily upgradable in view of novel discoveries. Moreover, compared with whole exome sequencing, analysis of the multigene panel is easier and faster. Overall, we identified 8 disease-causing variants in 5 different genes (NIPAL4, TGM1, CYP4F22, $A B C A 12$ and $C E R S 3)$. Among these, three were novel (c.118C $>$ T in NIPAL4; c.5898G $>C$ and c. $2855 \mathrm{~A}>\mathrm{G}$ in $A B C A 12)$. Using bioinformatic tools, these variations were predicted as pathogenic and causing a structural protein modification suggesting their involvement in ichthyosis. Moreover, already described mutations were pathogenic according to ClinVar database, except for c.844 $\mathrm{C}>\mathrm{T}$ in CYP4F22 gene which was classified as an uncertain significant variant. However, this variant was associated to both LI and CIE phenotypes [29, 30]. Moreover, c.845G > A (p.Arg282Gln) variant which is closed to c.844 $\mathrm{C}>\mathrm{T}$ variation, was classified as likely pathogenic in ClinVar arguing the pathogenic effect of this mutation.

Unlike ARCI series in literature, where TGM1 was the first responsible gene [31-33], in our cohort NIPAL4 was rather the most involved gene (36\%) with two missense mutations (c.534A $>\mathrm{C}$ and c. $118 \mathrm{~T}>\mathrm{C}$ ). This discordance could be explained by the small number of patients investigated in our study.

Phenotype-genotype correlation was one of the major objectives of the current investigation. Besides finding new variations in genes causing ichthyosis, we tried to highlight novel clinical characteristics associated with particular genotypes. In this context, a particular form of non-syndromic ichthyosis (ILC) with multiple localized polycyclic erythematous and squamous plaques and brownish fine scales was found in patients carrying two novel missense mutations in $A B C A 12$ gene. These variations and clinical related aspects have never been reported before. In fact, this gene is involved in either HI phenotype [34], with homozygous or compound heterozygous truncating mutations, or in CIE/LI with missense mutations [35-37]. On the other hand, ILC is quite different from Netherton syndrome which associates ILC with a specific hair-shaft abnormality and rather caused by mutations in SPINK5 gene [38]. As clearly observed, no pathogenic variations in SPINK5 gene, neither in genes involved in erythrokeratoderma variabilis (EKV) were found in ILC patients (data not shown), confirming then different etiologies. Recently, Leersum et al. [39] also reported a novel clinical phenotype of ichthyosis in a 3-year-old girl presenting a linear erythematosquamous lesions following the lines of Blaschko caused by ABCA12 mosaicism. This particular form of ichthyosis was explained by the combination of a germline mutation and an acquired postzygotic mutation in this gene. $A B C A 12$ gene was reported also in a keratosis pilaris with missense mutation and in Nevus comedonicus with a high protein expression level in the sebaceous gland without any variation in $A B C A 12$ coding region [40].

Otherwise, our investigation showed that $75 \%$ of our patients with NIPAL4 mutations presented with a phenotype of non-syndromic CIE. Concerning patients with TGM1 mutations, $66.67 \%$ of them showed a phenotype of LI. These observations confirmed other studies showing that NIPAL4 mutations were usually associated with a moderate phenotype with fine grey/white scales, while TGM1 mutations were associated with a more severe phenotype with darker, adherent and plate-like scales [41].

Our findings showed that some clinical features were associated with particular genotypes (Table 4). Thus; we noted brachydactyly in all cases mutated in TGM1 gene. This phenotype with 'long palm/short fingers' appearance was also reported in two Tunisian patients with LI carrying TGM1 mutations [42]. Even though not reported

Table 4 Clinical features and associated genes in studied ichthyosis patients

\begin{tabular}{lll}
\hline Gene & Clinical feature & Phenotype \\
\hline NIPAL4 & Collodion baby, Ectropion, Folds involvement, Palmoplantar hyperkeratosis & $\mathrm{CIE}, \mathrm{LI}$ \\
TGM1 & Brachydactyly, Collodion baby, Ectropion, Folds involvement, Palmoplantar hyperkeratosis, Ears & $\mathrm{CIE}, \mathrm{LI}$ \\
CYP4F22 & malformation, Alopecia & $\mathrm{LI}$ \\
CERS3(ADAM17) & Collodion baby, Palmoplantar hyperkeratosis & $\mathrm{CIE} \cdot$ \\
\hline
\end{tabular}

CIE congenital ichthyosiform erythoderma; L/ lamellar ichthyosis; CIE CIE with ocular defect 
in the literature, we suggest considering brachydactyly as indicative of TGM1 mutations. To the best of our knowledge, this phenotype, noted in E1 patient also, has never been reported before in ARCI patients with CERS3 mutations. We suggest that this symptom may be caused by the deletion of ADAMTS17 gene [43]. Indeed, a founder homozygous contiguous gene deletion (including exon 13 of CERS3, complete sequence of non-coding RNA FLJ42289 and the first three exons of ADAMTS17) was reported in three consanguineous Tunisian families affected with ichthyosis associated with ocular, cardiac and skeletal anomalies [44]. Since E1 patient presented a similar skin phenotype with eye anomaly, and came from the same area, it was very likely that she carried the same described deletion [44]. In order to confirm this hypothesis, further analyses would be necessary.

In our cohort, 7 patients were born as collodion babies and were mutated either in TGM1 (3/7), NIPAL4 (3/7), or CYP4F22 (1/7) genes. In the literature, this clinical feature was significantly associated with TGM1 [41], and NIPAL4 mutations with varying frequencies (28$73 \%)[41,45]$. But it was also reported in patients carrying mutations in $A B C A 12, C E R S 3, A L O X 12 B, A L O X E 3$, CYP4F22, lipase N, PNPLA1 and SDR9C7 genes [46, 47].

Ectropion was noted in all studied patients with TGM1 mutations, and $50 \%$ of those with NIPAL4 mutations. This finding was consistent with literature data and confirmed the previous observation that ARCI patients bearing TGM1 mutations were more likely to develop bilateral ectropion [47].

In our study, skin folds were spared in 2 patients with NIPAL4 variation and in the patient with CYP4F22 variation. Although reported in the literature [48] as a predilection site to be involved, large skin folds when spared could not rule out variations in NIPAL4 and CYP4F22 genes.

Our findings showed that palmoplantar keratoderma was associated with NIPAL4, TGM1, CYP4F22 mutations and CERS3 deletion, but with varying severity. Thus, in patients with p.Met63Thr NIPAL4 variation PPK was either moderate or absent. However, PPK was rather yellowish and severe in patients with p.Glu178 Asp NIPAL4 mutation as reported in the literature. Indeed, yellowish PPK has been reported in NIPAL4 series and, to the best of our knowledge, never associated with other ARCI genes [45]. Thus, yellowish keratoderma could be indicative of NIPAL4 mutation in ARCI patients. In addition, patient $\mathrm{C} 1$ showed a constricting band around his right fifth finger, suggestive of pseudoainhum (Fig. 1b). The latter is a rare complication of ARCI and several other disorders of keratinization such as Vohwinkel syndrome and Mal de Meleda [49]. It has been suggested that pseudoainhum may be the consequence of repetitive infections or constriction by a thick plate of keratin [49]. However, the exact pathogenic mechanism in ARCI remains unclear.

In our TGM1 mutated patients, we reported mainly a hyperlinearity of the palms and soles, with sometimes a severe PPK.

Ears deformity was reported in two patients (B1 andY1) and alopecia in one patient (M1) carrying TGM1 mutation. Ears deformity was more commonly found in TGM1, ABCA12, ALOX12B and ALOXE3 mutations compared to other ARCI forms [41]. Alopecia was found to be significantly associated with TGM1 mutations.

Nevertheless, we also revealed that diverse phenotypes were caused by the same gene with an identical mutation. Notably, c.788G > A in TGM1 gene was found in both CIE (M1 patient) and LI (B1 patient), suggesting the effect of a modifier gene as it was already reported [50]. In an attempt to explain this phenotypic heterogeneity, we examined $F L G$ gene coding the filaggrin protein. We identified 44 missense polymorphisms in M1 not present in B1 (data not shown). Prediction effect of these polymorphisms showed that the majority were benign.

To summarize, we report three novel mutations, two of which were located in ABCA12 gene responsible for ILC, a rare clinical form of ichthyosis, as well as novel clinical characteristics associated with particular genotypes.

\section{Conclusions}

Phenotypic-genotypic correlation suggests that brachydactyly could be related to TGM1 mutations and associated to deletion of ADAMTS17 gene associated to deletion of CERS3 exon 13.

Besides the new reported variations in NIPAL4 and $A B C A 12$ genes, we added new insights to the already reported particular phenotypes linked to specific genes. The involvement of such genes in a particular ARCI form remains discussed. In order to better explain its phenotype heterogeneity, investigation of whole genome is necessary to search for responsible modifier genes.

\section{Web resources \\ http://bioinfo.ut.ee/primer3-0.4.0/ \\ http://www.ncbi.nlm.nih.gov/blast/ \\ https://www.genome.jp/tools-bin/clustalw \\ http://genetics.bwh.harvard.edu/pph2/ \\ https://sift.bii.a-star.edu.sg/ \\ http://www.mutationtaster.org/ \\ http://www.pantherdb.org/tools/index.jsp \\ https://cadd.gs.washington.edu/snv}




\section{Abbreviations}

ARCI: Autosomal recessive congenital ichthyosis; ILC: Ichthyosis linearis circumflexa; CIE: Congenital ichthyosiform erythroderma; LI: Lamellar ichthyosis; HI: Harlequin ichthyosis; NGS: Next generation sequencing.

\section{Acknowledgements}

We gratefully thank all family members of the participants for their valuable cooperation and for providing the blood samples. We also thank all the members of the department of dermatology University Hospital- Sfax-Tunisia. This study was funded by The Ministry of Higher Education and Scientific ResearchTunisia (LR16E16). Our thanks are also addressed to Mr. Mohamed Sami Aifa for his critical reading of this paper and Mr Kamel Maaloul for proofreading the manuscript.

\section{Authors' contributions}

ME participated in the practical and theoretical part of the article, ADZ: extracted the raw data of the panel, EB participated in the recruitment of patients, RC: participated in the recruitment of patients and medical theoretical part of the article, SM: participated in the recruitment of patients and medical theoretical part of the article, HT: participated in the recruitment of patients, FF: participated in the practical part of the article, NB-E participated in the practical and theoretical part of the article, JF participated in the practical part of the article. All authors read and approved the final manuscript.

\section{Funding}

Ministry of higher education and scientific research Tunisia.

\section{Availability of data and materials}

The datasets used and/or analyzed during the current study are available in NCBI SRA at https://dataview.ncbi.nlm.nih.gov/object/PRJNA765516?revie wer=pu6eruli4285rvodofg5o2qndc.

\section{Declarations}

\section{Ethical approval and consent to participate}

The study protocol conforms to the approval of local ethical committee of CHU Hedi Chaker Sfax, in compliance with the Declaration of Helsinki. Written informed consent was obtained from all study participants or the parents for minor children.

\section{Consent for publication}

All participants provided informed consent and they agreed to publish their clinical data.

\section{Competing interests}

The authors declare no competing interests.

\section{Author details}

${ }^{1}$ Laboratory of Molecular and Functional Genetics, Faculty of Sciences of Sfax, Sfax University, Sfax University, Street of Soukra km 4, BP 1171-3000, Sfax, Tunisia. ${ }^{2}$ Faculty of Medicine, Institute of Human Genetics, Medical Center, University of Freiburg, Freiburg, Germany. ${ }^{3}$ Department of Dermatology, CHU Hedi Chaker, Sfax University, Sfax, Tunisia.

\section{Received: 9 September 2021 Accepted: 31 December 2021}

Published online: 05 January 2022

\section{References}

1. Richard G. Molecular genetics of the ichthyoses. Am J Med Genet C Semin Med Genet. 2004;131C:32-44.

2. Traupe H, Fischer J, Oji V. Non syndromic types of ichthyosis - an update. J Dtsch Dermatol Ges. 2014;12:109-21.

3. Oji V, Tadini G, Akiyama M, Blanchet Bardon C, Bodemer C, Bourrat E, et al. Revised nomenclature and classification of inherited ichthyoses: results of the First Ichthyosis Consensus Conference in Sorèze 2009. Am Acad Dermatol. 2010:63:607-41.
4. Williams ML, Elias PM. Genetically transmitted, generalized disorders of cornification: the ichthyoses. Dermatol Clin. 1987;5:155-78.

5. Akiyama M, Sawamura D, Shimizu H. The clinical spectrum of nonbullous congenital ichthyosiform erythroderma and lamellar ichthyosis. Clin Exp Dermatol. 2003:28:235-40.

6. Wajid M, Kurban M, Shimomura Y, Christiano AM. NIPAL4/ichthyin is expressed in the granular layer of human epidermis and mutated in two Pakistani families with autosomal recessive ichthyosis. Dermatology (Basel). 2010:220:8-14.

7. Russell LJ, DiGiovanna JJ, Rogers GR, Steinert PM, Hashem N, Compton $J G$, et al. Mutations in the gene for transglutaminase 1 in autosomal recessive lamellar ichthyosis. Nat Genet. 1995:9:279-83.

8. Lefèvre C, Bouadjar B, Karaduman A, Jobard F, Saker S, Ozguc M, et al. Mutations in ichthyin a new gene on chromosome 5 q33 in a new form of autosomal recessive congenital ichthyosis. Hum Mol Genet. 2004;13:2473-82.

9. Krebsová A, Küster W, Lestringant GG, Schulze B, Hinz B, Frossard PM, et al. Identification, by homozygosity mapping, of a novel locus for autosomal recessive congenital ichthyosis on chromosome $17 p$, and evidence for further genetic heterogeneity. Am J Hum Genet. 2001;69:216-22.

10. Kelsell DP, Norgett EE, Unsworth H, Teh M-T, Cullup T, Mein CA, et al. Mutations in ABCA12 underlie the severe congenital skin disease harlequin ichthyosis. Am J Hum Genet. 2005;76:794-803.

11. Shigehara Y, Okuda S, Nemer G, Chedraoui A, Hayashi R, Bitar F, et al. Mutations in SDR9C7 gene encoding an enzyme for vitamin A metabolism underlie autosomal recessive congenital ichthyosis. Hum Mol Genet. 2016:25:4484-93.

12. Toribio J, Fernández Redondo V, Peteiro C, Zulaica A, Fabeiro JM. Autosomal dominant lamellar ichthyosis. Clin Genet. 1986;30:122-6.

13. Heinz L, Kim G-J, Marrakchi S, Christiansen J, Turki H, Rauschendorf M-A, et al. Mutations in SULT2B1 Cause Autosomal-Recessive Congenital Ichthyosis in Humans. Am J Hum Genet. 2017;100:926-39.

14. Virolainen E, Wessman M, Hovatta I, Niemi KM, Ignatius J, Kere J, et al. Assignment of a novel locus for autosomal recessive congenital ichthyosis to chromosome 19p13.1-p13.2. Am J Hum Genet. 2000;66:1132-7.

15. Eckl K-M, Tidhar R, Thiele H, Oji V, Hausser I, Brodesser S, et al. Impaired epidermal ceramide synthesis causes autosomal recessive congenital ichthyosis and reveals the importance of ceramide acyl chain length. J Invest Dermatol. 2013;133:2202-11.

16. Jobard F, Lefèvre $C$, Karaduman $A$, Blanchet-Bardon $C$, Emre S, Weissenbach J, et al. Lipoxygenase-3 (ALOXE3) and 12(R)-lipoxygenase (ALOX12B) are mutated in non-bullous congenital ichthyosiform erythroderma (NCIE) linked to chromosome 17p13.1. Hum Mol Genet. 2002;11:107-13.

17. Israeli S, Khamaysi Z, Fuchs-Telem D, Nousbeck J, Bergman R, Sarig O, et al. A mutation in LIPN, encoding epidermal lipase $\mathrm{N}$, causes a late-onset form of autosomal-recessive congenital ichthyosis. Am J Hum Genet. 2011:88:482-7.

18. Klar J, Schweiger M, Zimmerman R, Zechner R, Li H, Törmä H, et al. Mutations in the fatty acid transport protein 4 gene cause the ichthyosis prematurity syndrome. Am J Hum Genet. 2009;85:248-53.

19. Gg L, W K, Pm F, R H. Congenital ichthyosis, follicular atrophoderma, hypotrichosis, and hypohidrosis: a new genodermatosis? Am J Med Genet. 1998;75:186-9.

20. Vahlquist A, Törmä H. Ichthyosis: a road model for skin research. Acta Derm Venereol. 2020;100:197-206.

21. Lewin HA, Stewart-Haynes JA. A simple method for DNA extraction from leukocytes for use in PCR. Biotechniques. 1992;13:522-4.

22. Li H, Durbin R. Fast and accurate short read alignment with BurrowsWheeler transform. Bioinformatics. 2009;25:1754-60.

23. DePristo MA, Banks E, Poplin R, Garimella KV, Maguire JR, Hartl C, et al. A framework for variation discovery and genotyping using next-generation DNA sequencing data. Nat Genet. 2011;43:491-8.

24. Garrison E, Marth G. Haplotype-based variant detection from short-read sequencing. arXiv: arXiv:1207.3907 [q-bio]. 2012.

25. Lai Z, Markovets A, Ahdesmaki M, Chapman B, Hofmann O, McEwen $\mathrm{R}$, et al. VarDict: a novel and versatile variant caller for next-generation sequencing in cancer research. Nucleic Acids Res. 2016;44:e108.

26. Li H. A statistical framework for SNP calling, mutation discovery, association mapping and population genetical parameter estimation from sequencing data. Bioinformatics. 2011;27:2987-93. 
27. Wang K, Li M, Hakonarson H. ANNOVAR: functional annotation of genetic variants from high-throughput sequencing data. Nucleic Acids Res. 2010;38:e164.

28. McLaren W, Gil L, Hunt SE, Riat HS, Ritchie GRS, Thormann A, et al. The ensembl variant effect predictor. Genome Biol. 2016;17:122.

29. Feng $C$, Wang $H$, Lee $M$, Zhao J, Lin Z, Yang Y. Two missense mutations in CYP4F22 in autosomal recessive congenital ichthyosis. Clin Exp Dermatol. 2017;42:98-100

30. Bučková H, Nosková H, Borská R, Réblová K, Pinková B, Zapletalová E, et al. Autosomal recessive congenital ichthyoses in the Czech Republic. Br J Dermatol. 2016;174:405-7.

31. Fischer J. Autosomal recessive congenital ichthyosis. J Investig Dermatol. 2009;129:1319-21.

32. Israeli S, Goldberg I, Fuchs-Telem D, Bergman R, Indelman M, BittermanDeutsch O, et al. Non-syndromic autosomal recessive congenital ichthyosis in the Israeli population. Clin Exp Dermatol. 2013;38:911-6.

33. Youssefian L, Vahidnezhad H, Saeidian AH, Touati A, Sotoudeh S, Mahmoudi $\mathrm{H}$, et al. Autosomal recessive congenital ichthyosis: genomic landscape and phenotypic spectrum in a cohort of 125 consanguineous families. Hum Mutat. 2019;40:288-98.

34. Takeichi T, Akiyama M. Inherited ichthyosis: Non-syndromic forms. J Dermatol. 2016;43:242-51.

35. Akiyama M. ABCA12 mutations and autosomal recessive congenital ichthyosis: a review of genotype/phenotype correlations and of pathogenetic concepts. Hum Mutat. 2010;31:1090-6.

36. Lefévre C, Audebert S, Jobard F, Bouadjar B, Lakhdar H, BoughdeneStambouli $\mathrm{O}$, et al. Mutations in the transporter ABCA12 are associated with lamellar ichthyosis type 2. Hum Mol Genet. 2003;12:2369-78.

37. Natsuga K, Akiyama M, Kato N, Sakai K, Sugiyama-Nakagiri Y, Nishimura $M$, et al. Novel ABCA12 mutations identified in two cases of non-bullous congenital ichthyosiform erythroderma associated with multiple skin malignant neoplasia. J Investig Dermatol. 2007;127:2669-73.

38. Sarri CA, Roussaki-Schulze A, Vasilopoulos Y, Zafiriou E, Patsatsi A, Stamatis $C$, et al. Netherton syndrome: a genotype-phenotype review. Mol Diagn Ther. 2017:21:137-52.

39. van Leersum FS, Seyger MMB, Theunissen TEJ, Bongers EMHF, Steijlen PM, van Geel M. Recessive mosaicism in ABCA12 causes blaschkoid congenital ichthyosiform erythroderma. Br J Dermatol. 2020;182:208-11.

40. Liu F, Yang Y, Zheng Y, Liang Y-H, Zeng K. Mutation and expression of ABCA12 in keratosis pilaris and nevus comedonicus. Mol Med Rep. 2018;18:3153-8.

41. Simpson JK, Martinez-Queipo M, Onoufriadis A, Tso S, Glass E, Liu L, et al. Genotype-phenotype correlation in a large English cohort of patients with autosomal recessive ichthyosis. Br J Dermatol. 2020;182:729-37.

42. Louhichi N, Hadjsalem I, Marrakchi S, Trabelsi F, Masmoudi A, Turki H, et al. Congenital lamellar ichthyosis in Tunisia is caused by a founder nonsense mutation in the TGM1 gene. Mol Biol Rep. 2013;40:2527-32.

43. Oichi T, Taniguchi Y, Soma K, Oshima Y, Yano F, Mori Y, et al. Adamts 17 is involved in skeletogenesis through modulation of BMP-Smad1/5/8 pathway. Cell Mol Life Sci. 2019;76:4795-809.

44. Radner FPW, Marrakchi S, Kirchmeier P, Kim G-J, Ribierre F, Kamoun B, et al Mutations in CERS3 Cause Autosomal Recessive Congenital Ichthyosis in Humans. PLOS Genet. 2013;9:e1003536.

45. Alavi A, Shahshahani MM, Klotzle B, Fan J-B, Ronaghi M, Elahi E. Manifestation of diffuse yellowish keratoderma on the palms and soles in autosomal recessive congenital ichthyosis patients may be indicative of mutations in NIPAL4. J Dermatol. 2012;39:375-81.

46. Sugiura K, Akiyama M. Update on autosomal recessive congenital ichthyosis: mRNA analysis using hair samples is a powerful tool for genetic diagnosis. J Dermatol Sci. 2015;79:4-9.

47. Farasat S, Wei M-H, Herman M, Liewehr DJ, Steinberg SM, Bale SJ, et al. Novel transglutaminase-1 mutations and genotype-phenotype investigations of 104 patients with autosomal recessive congenital ichthyosis in the USA. J Med Genet. 2009;46:103-11.

48. Ballin N, Hotz A, Bourrat E, Küsel J, Oji V, Bouadjar B, et al. Genetical, clinical and functional analysis of a large international cohort of patients with autosomal recessive congenital ichthyosis due to mutations in NIPAL4. Hum Mutat. 2019. https://doi.org/10.1002/humu.23883.

49. Behera B, Chandrashekar L, Singh N, Thappa DM, Gochhait D. Lamellar ichthyosis associated bilateral pseudoainhum of fingers and toes successfully treated with tazarotene. Dermatol Ther. 2017;30.
50. Li H, Loriè EP, Fischer J, Vahlquist A, Törmä $H$. The expression of epidermal lipoxygenases and transglutaminase- 1 is perturbed by NIPAL4 mutations: indications of a common metabolic pathway essential for skin barrier homeostasis. J Invest Dermatol. 2012;132:2368-75.

51. Esposito G, Rescigno G, Salvatore F, Auricchio L, Paparo F, Rinaldi M. Transglutaminase 1 gene mutations in Italian Patients with autosomal recessive lamellar ichthyosis. J Investig Dermatol. 2001;116:809-12.

52. Rodríguez-Pazos L, Ginarte M, Vega A, Toribio J. Autosomal recessive congenital ichthyosis. Actas Dermosifiliogr. 2013;104:270-84.

53. Liu J-J, Yuan Y-Y, Zhang X-Q, Li Z-M, Xu Y-S, Gao S-M, et al. Mutations of transglutaminase-1 in Chinese patients with autosomal recessive congenital ichthyosis: a case report with clinical and genetic analysis of Chinese cases reported in literature. Clin Exp Dermatol. 2015;40:56-62.

\section{Publisher's Note}

Springer Nature remains neutral with regard to jurisdictional claims in published maps and institutional affiliations.
Ready to submit your research? Choose BMC and benefit from:

- fast, convenient online submission

- thorough peer review by experienced researchers in your field

- rapid publication on acceptance

- support for research data, including large and complex data types

- gold Open Access which fosters wider collaboration and increased citations

- maximum visibility for your research: over $100 \mathrm{M}$ website views per year

At BMC, research is always in progress.

Learn more biomedcentral.com/submissions 